\title{
An Open Pilot Trial of Written Exposure Therapy for Patients With Post-Traumatic Stress Disorder in Korea
}

\author{
Ju-Eun Park', Kyeong-Sook Choi ${ }^{1,2}$, Yu-Ri Han ${ }^{3}$, Jeong-Eun Kim¹, \\ Jay Song ${ }^{1}$, Je-Chun $\mathrm{Yu}^{1,2}$, and Ji-Ae Yun ${ }^{1,2} \bowtie$ \\ ${ }^{1}$ Department of Neuropsychiatry, Daejeon Eulji Medical Center, Daejeon, Republic of Korea \\ ${ }^{2}$ Department of Neuropsychiatry, Eulji University School of Medicine, Daejeon, Republic of Korea \\ ${ }^{3}$ Yonsei University Wonju College of Medicine, Wonju, Republic of Korea
}

\begin{abstract}
Objective Written exposure therapy (WET) is exposure therapy for post-traumatic stress disorder (PTSD). Compared to evidencebased treatments for PTSD, WET requires only five sessions, has a shorter session time, and no between-session assignments. The current study examined the efficacy of WET among Korean patients with PTSD due to various traumatic events on PTSD symptoms, depressive symptoms, and global functioning levels.

Methods The study recruited 41 patients with a current primary diagnosis of PTSD in psychiatric outpatient clinics. Assessments were conducted at baseline, and at 6,12, and 24 weeks following the first treatment session.

Results In total, 25 patients started WET. Findings showed a significant reduction in the rate of PTSD diagnosis and symptom severity scores. Fourteen of $23(60.9 \%)$ patients at 6 weeks, 15 of $22(68.2 \%)$ patients at 12 weeks, and 14 of $18(77.8 \%)$ patients at 24 weeks no longer met the diagnosis of PTSD. Depressive symptoms and global function scores also improved after WET. The dropout rate was $8 \%$ $(\mathrm{n}=2)$.

Conclusion This study suggests the feasibility of implementing WET among various types of patients with PTSD in Korea and other Asian countries.

Psychiatry Investig 2021;18(8):728-735
\end{abstract}

Key Words Written disclosure, Exposure therapy, Asians, Koreans, Global function.

\section{INTRODUCTION}

Post-traumatic stress disorder (PTSD) is a highly distressing and debilitating disorder. ${ }^{1}$ Numerous studies on the effects of PTSD have consistently shown various disabilities in functional aspects, such as occupational impairment, ${ }^{2}$ unemployment and low income, ${ }^{3,4}$ interpersonal problems, ${ }^{5,6}$ and high psychiatric $^{7}$ and medical comorbidities. ${ }^{8}$ Thus, chronic PTSD represents a significant public health problem.

To address this public health threat, some evidence-based treatments (EBT) for PTSD have been developed, and their effectiveness has been demonstrated. These include trauma-fo-

\footnotetext{
Received: April 2, 2021 Revised: May 11, 2021

Accepted: May 23, 2021

$\square$ Correspondence: Ji-Ae Yun, MD

Department of Neuropsychiatry, Daejeon Eulji Medical Center, 95 Dunsanseo-ro, Seo-gu, Daejeon 35233, Republic of Korea

Tel: +82-42-611-3443, Fax: +82-42-611-3445, E-mail: jayun5959@gmail.com (c) This is an Open Access article distributed under the terms of the Creative Commons Attribution Non-Commercial License (https://creativecommons.org/licenses/bync/4.0) which permits unrestricted non-commercial use, distribution, and reproduction in any medium, provided the original work is properly cited.
}

cused cognitive behavioral therapy, cognitive processing therapy (CPT), and prolonged exposure therapy (PE). These are strongly recommended first-line therapies in major PTSD treatment guidelines. ${ }^{9}$

Recently, Sloan and Marx's ${ }^{10}$ research team introduced a fivesession exposure-based PTSD treatment protocol called written exposure therapy (WET). WET is recommended for the treatment of PTSD in clinical practice guidelines (Department of Veterans Affairs/Department of Defense, 2017). Like other EBTs, it comprises critical components considered to be responsible for the successful treatment of PTSD symptoms, such as therapeutic exposure and cognitive processing/cognitive restructuring. ${ }^{10-12}$ However, WET requires a smaller number of sessions, shorter session durations, and no between-session assignments. $^{10,13}$

The efficacy of WET was evaluated using a randomized controlled trial, and this study reported a significant reduction in PTSD symptom severity at the post-treatment follow-up, and symptom reduction was maintained three and six months later with $7 \%$ of drop-out rate. ${ }^{14}$ A study that directly compared WET 
to CPT indicated that WET was non-inferior to CPT. ${ }^{15}$ Additionally, WET resulted in substantially lower rates of treatment dropout compared to CPT (6\% vs. $39 \%$ ). ${ }^{15}$ The effects of treatment were also long-term, specifically lasting for 60 weeks. ${ }^{16,17}$

In order to apply WET to Korean patients, we must consider their different cultural background, which is dissimilar to Westerners. According to a study conducted in Japan, which is culturally similar to Korea, only $15 \%$ of respondents choose to disclose their trauma. ${ }^{18}$ This tendency to be reluctant to express and verbalize trauma might be a barrier to exposure therapy. However, PE identical to the original version yielded the same effectiveness as western studies in Japanese studies. ${ }^{19,20}$ Furthermore, Korean culture has been influenced by Western cultures in some way, such that they share a few basic assumptions. ${ }^{21}$ These common aspects are expected to help in successfully applying WET to Korean patients without a need to modify the protocols. Therefore, further evidence is needed regarding the applicability of WET to different population groups.

We aimed to address this issue by implementing WET on Korean patients with PTSD. Subsequently, we compared the participants' PTSD symptoms, depressive symptoms, and global functioning levels before and after treatment.

\section{METHODS}

\section{Participants}

Patients suspected of having PTSD were referred from psychiatric outpatient clinics between March 2019 to November 2020. The subjects were recruited by five psychiatrists, who recommended them to participate in the study. Participants who agreed to participate provided written consent. If the participant was an adolescent, we also explained the treatment to their parents and obtained written consent. The study was approved by the Institutional Review Boards at Eulji University Hospital (EMC 2019-01-008-005).

Inclusion criteria was a PTSD diagnosis as determined by the Clinician-Administered PTSD Scale for DSM-5 (CAPS-5). Exclusion criteria comprised the presence of a recent manic episode or psychotic disorder, present alcohol and other substance dependence, current suicidal ideation severe enough to warrant immediate intervention, other psychiatric disorders severe enough to warrant designation as the primary disorder, or current engagement in EBT for PTSD.

All participants underwent WET while continuing their usual psychiatric treatment and medication regimen with their own doctor. In total, 23 participants who underwent WET were monitored for medication use up to 6 weeks following the end of treatment. Of the 23 participants, 19 (82.6\%) had Selective Serotonin Reuptake Inhibitors (SSRIs), 2 (8.7\%) had non-SSRI antidepressants, and $2(8.7 \%)$ had two antidepressant combi- nations. Antipsychotics augmentation was performed on seven participants (30.4\%), and the dose of medication was used as the augmentation regimen below the therapeutic dosage. Of the 23 participants, $30.4 \%$ had never used Benzodiazepine. Only one participant who started antipsychotics augmentation during WET changed medications. Four participants had experienced EBT for PTSD, three were receiving stabilization therapy, and one participant was undergoing PE for other index trauma.

\section{Measures}

Clinical interviews at baseline were conducted by five psychiatrists who had at least a master's degree in psychiatry. The Structured Clinical Interview for DSM-5 (SCID) was used to assess baseline psychiatric comorbidities and PTSD diagnosis.

Assessments of PTSD diagnostic status and symptom severity using the CAPS and Global Assessment of Function (GAF) score were conducted at baseline. Follow-up assessments were also conducted at 6 and 12 weeks following the first treatment session. Assessment points were selected based on a previous similar study to ensure comparable results. ${ }^{15}$ Two clinical psychologists who are currently working in the department of psychiatry in university hospital conducted CAPS and were not informed of the treatment conditions. They both held a master's degree in psychology, and have been certified as clinical psychologist specialists and level-one mental health clinical psychologists in Korea.

Sociodemographic characteristics, trauma type, age at the time of trauma exposure, and history of past EBT for PTSD were obtained via questionnaires at baseline. According to previous studies, ${ }^{15,22}$ trauma types were divided into five categories for analysis purposes. These were: 1 ) child/adult sexual assault (including rape, sexual assault other than rape, being stalked, or physical abuse by a romantic partner); 2) physical violence; 3 ) accidents (including life-threatening automobile accidents, other life-threatening accidents, accidentally injuring/killing someone); 4) the unexpected or traumatic death of a loved one; and 5) other traumas of loved ones or witnessing traumas.

Self-reported questionnaires for PTSD (PTSD check List-5, PCL-5) and depression (Patient Health Questionnaire-9, PHQ9) were conducted at baseline and follow-up. We also assessed participants' beliefs about the expected outcome of treatment at the end of the first treatment session using the Treatment Expectancy Questionnaire (TEQ). We further assessed treatment satisfaction at the last treatment session using the Client Service Questionnaire.

Clinician-Administered PTSD Scale for DSM-5 (CAPS-5)

The CAPS- 5 is a 30 -item structured interview that can be used to make a current (in the past month) diagnosis of PTSD. ${ }^{23}$ 
The 30-item questionnaire corresponds to the DSM-5 diagnosis for PTSD. The primary outcome was to achieve improvements in CAPS-5 PTSD symptom severity and the diagnostic status of PTSD. Diagnostic status was determined based on the DSM- 5 algorithm. The Korean version of the CAPS- 5 has good reliability and validity.

\section{PTSD checklist-5 (PCL-5)}

The PCL-5 is a self-rated inventory developed for screening PTSD symptoms based on the DSM- 5 criteria. It includes 20 items. ${ }^{25}$ Each item has five response options, with scores ranging from 0 to 4 . The total score on the PCL-5 scale ranges from 0 to 80 . The Korean version of the PCL- 5 has good psychometric properties. ${ }^{26}$

\section{Patient Health Questionnaire-9 (PHQ-9)}

The PHQ-9 is an inventory used to screen, diagnose, monitor, and measure the severity of depression. The PHQ-9 includes 9 questions to rate the frequency of symptoms according to a scoring severity index ranging from 0 to 3 . The total score ranges from 0 to 27. The Korean version of the PHQ-9 has good psychometric properties. ${ }^{27}$

\section{Global assessment of function (GAF)}

The GAF is an inventory used by mental health clinicians and physicians to subjectively rate the social, occupational, and psychological functioning of an individual. The score ranges from 1 ("severely impaired") to 100 ("extremely high functioning").28

\section{Treatment expectancy questionnaire (TEQ)}

As in previous studies, ${ }^{14,15,29}$ treatment credibility was evaluated using the $\mathrm{TEQ}^{30}$ after the first session. This scale asks participants about how logical the treatment seems, their confidence in undergoing the treatment and recommending it to others, and their expectations of the treatment's success. We translated the TEQ into Korean and used the adapted version. The Cronbach's alpha for the TEQ was 0.791 in the present study. This measure utilizes two rating scales, one ranging from 1 to 9 , and another from $0 \%$ to $100 \%$. Therefore, we standardized each item and formed a composite for each factor. ${ }^{31}$

\section{Client Service Questionnaire (CSQ-8)}

As in previous studies, ${ }^{14,15,29}$ we measured satisfaction with the WET at the last session before concluding the treatment, using the CSQ-8. ${ }^{32}$ The CSQ-8 has eight items rated on a 4-point Likert-type scale, ranging from 8 to 32 . Higher scores indicate greater satisfaction. We translated the CSQ- 8 into Korean and used the adapted version. The Cronbach's alpha for the CSQ-8 was 0.871 in the present study.

\section{Treatment}

The WET comprised 5 weekly sessions, with each WET session lasting approximately 45 minutes. All sessions followed the WET protocol proposed by Sloan and Marx ${ }^{10}$ with one exception. Although the WET protocol proposed by Sloan suggests that psychoeducation be conducted in the first session, we performed psychoeducation for about one hour during the pre-session before commencing WET, to allow sufficient time for psychoeducation. Thus, the WET protocol used in our study consisted of five 45-minute weekly sessions, with the pre-session lasting one hour. During the pre-session, participants were provided with PTSD psychoeducation information and the WET treatment rationale.

At the beginning of each of the five treatment sessions, the therapist read the writing instructions to the participant. The participant conducted exposure through writing for $30 \mathrm{~min}$ utes alone in a separate room adjacent to the clinic room. To prepare for an unexpected emergency, they were informed to notify the therapist or press the bell attached to the desk in the treatment room if a participant needs help from a therapist during the writing.

After 30 minutes of writing, the check-in interview was conducted briefly for about 15 minutes. No other explicit betweensession homework was assigned to participants.

All sessions were conducted by a psychiatrist with a master's degree. The therapist had a year of supervised experience by a certified supervisor on two PE cases before this study.

Each session script included in the WET was manually written by the WET developer ${ }^{10}$ and initially translated by a bilingual psychiatrist and a psychologist. Subsequently, a back translation was done blindly by another bilingual psychiatrist. Eventually, an additional back translation was performed by a translation agency.

\section{Statistical analysis}

Paired samples t-tests were used to compare the psychiatric symptoms before and after treatment. A repeated measures analysis of variance (rmANOVA) was also performed to identify the symptom changes over three time points and calculate the effect size (i.e., partial $\mathrm{n}^{2}$ ). All statistical analyses were performed using IBM SPSS Statistics for Windows, version 21.0 (IBM Corp., Armonk, NY, USA).

\section{RESULTS}

A total of 43 patients were screened for PTSD using the SCID; of which, 41 participants were diagnosed with PTSD (Figure 1). One person was excluded because of seizure-like conversion symptoms, which was deemed unsuitable for exposure therapy. Six individuals were eligible but did not attend the baseline 


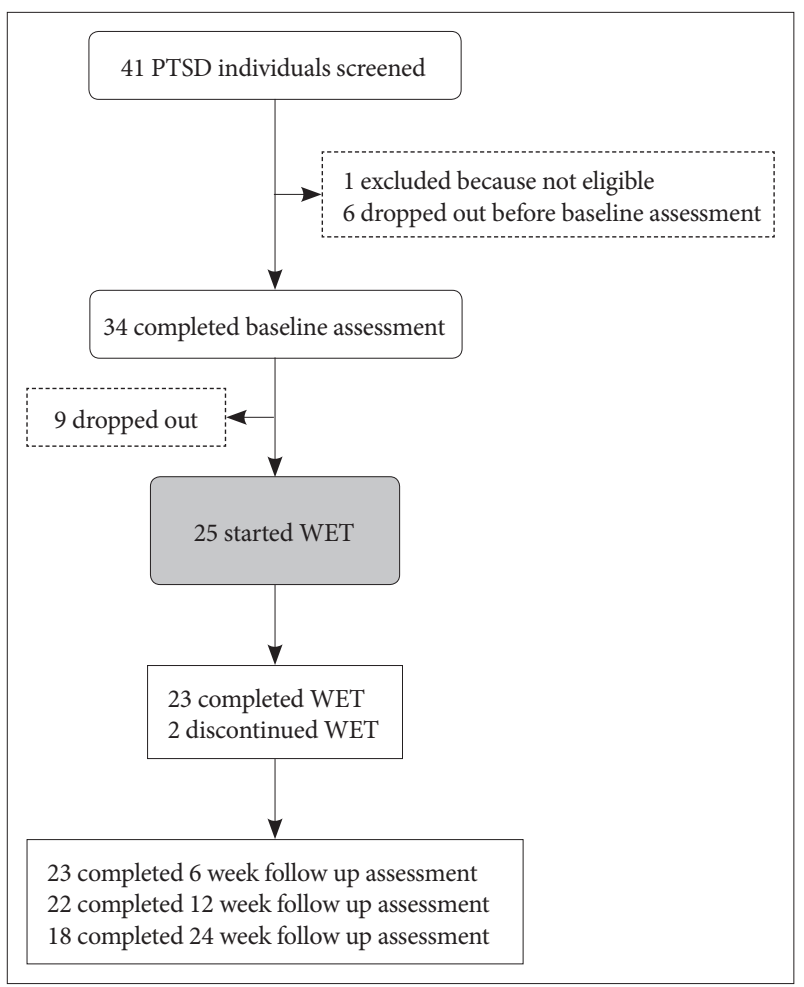

Figure 1. CONSORT diagram for study conducting WET for Korean participants with PTSD. PTSD: post-traumatic stress disorder, WET: written exposure therapy.

assessment. Thirty-four participants completed the baseline assessment. Table 1 shows the 34 participants' sociodemographic and clinical characteristics.

Of the 34 patients with PTSD who completed the baseline assessment, 14 (44.1\%) had a diagnosis of comorbid depression. The median PTSD duration was 14 months. Nine of the 34 participants dropped out spontaneously before commencing treatment. Ultimately, 25 people started WET. Five of the 25 participants had PTSD for less than 12 months. Among those who dropped out $(n=9)$, duration of PTSD was significantly shorter (14.56 \pm 27.14 vs. $43.72 \pm 41.15, \mathrm{t}=-2.385, \mathrm{p}=0.026)$ than participants who started WET. Socio-demographic factors and demographic factors did not differ significantly between the two groups.

Two of the 25 participants who started WET discontinued treatment at the first and second sessions, respectively. Both were women who had been sexually assaulted, one in childhood and one in adulthood. The remaining 23 participants completed five therapy sessions, as well as the 6- (23 of 23), 12(22 of 23), and 24-weeks (18 of 22) follow-up assessments. No severe adverse events were observed during the study.

Table 2 shows significant reductions in CAPS, PCL-5, and PHQ-9 scores after the treatment (6 weeks follow-up; T2), 12 weeks (T3), and 24 weeks (T4) follow-up. The GAF score also significantly increased after the treatment (T2, T3, and T4). At
Table 1. Demographic and clinical characteristics at baseline

\begin{tabular}{|c|c|c|}
\hline $\mathrm{N}=34$ & Mean $(\mathrm{N})$ & $\mathrm{SD}(\%)$ \\
\hline Age & 34.29 & 14.23 \\
\hline Sex (female) & 21 & 61.8 \\
\hline \multicolumn{3}{|l|}{ Marital status } \\
\hline Single & 20 & 58.8 \\
\hline Married & 8 & 23.5 \\
\hline Separated & 1 & 2.9 \\
\hline Divorced & 5 & 14.7 \\
\hline Education (year) & 11.56 & 3.57 \\
\hline \multicolumn{3}{|l|}{ Religion } \\
\hline Buddhism & 5 & 14.7 \\
\hline Catholic & 2 & 5.9 \\
\hline Christianity & 7 & 20.6 \\
\hline None & 20 & 58.8 \\
\hline \multicolumn{3}{|l|}{ Employment status } \\
\hline Full-time & 11 & 32.4 \\
\hline Part time & 3 & 8.8 \\
\hline Homemaker & 2 & 5.9 \\
\hline Student & 9 & 26.5 \\
\hline Unemployed & 9 & 26.5 \\
\hline \multicolumn{3}{|l|}{ Income (W10,000) } \\
\hline$\leq 100$ & 8 & 28.6 \\
\hline $100-300$ & 10 & 35.7 \\
\hline $300-500$ & 6 & 21.4 \\
\hline$\geq 500$ & 4 & 14.3 \\
\hline \multicolumn{3}{|l|}{ Trauma type } \\
\hline 1. Child sexual assault & 4 & 11.8 \\
\hline 2. Adult sexual assault & 13 & 38.2 \\
\hline 3. Physical violence & 7 & 20.6 \\
\hline 4. Accident & 9 & 26.5 \\
\hline 5. Unexpected traumatic death of loved one & 1 & 2.9 \\
\hline Diagnosis of comorbid depression at baseline & 14 & 44.1 \\
\hline
\end{tabular}

SD: standard deviation

6 weeks (T2), 14 of 23 (60.9\%) participants no longer met the PTSD diagnosis. Of the 23 participants, one did not complete the 12 weeks follow-up assessment. Among them, 15 (68.2\%) participants no longer met the PTSD diagnosis. Compared to the 6 weeks assessment, four additional participants did not meet the PTSD diagnosis at 12 weeks. Meanwhile, two participants who had shown improvements at 6 weeks were diagnosed with PTSD again at 12 weeks. At 24 weeks, four participants missed the follow-up assessment. Two of them had no PTSD diagnosis at the 12 weeks follow-up, and the other two showed no improvement at 12 weeks. At 24 weeks, 14 participants (77.8\%) no longer met the PTSD diagnosis. 
Table 2. Treatment outcomes of 5-week written exposure therapy for Korean patients with PTSD

\begin{tabular}{lccccc}
\hline $\mathrm{N}=25$ (2 dropped out) M (SD) & Baseline (T1) & Week 6 (T2) & Week 12 (T3) & Week 24 (T4) & Effect size (partial n $\left.{ }^{2}\right)$ \\
\hline CAPS-5\% free-diagnosis of PTSD & 0 of 25 (0) & 14 of 23 (60.9) & 15 of 22 (68.2) & 14 of 18 (77.8) & \\
CAPS-5 total score & $43.32(10.03)$ & $24.22(11.81)^{*}$ & $20.64(14.32)^{*}$ & $19.41(14.66)^{*}$ & 0.703 \\
PCL-5 & $52.56(13.29)$ & $31.04(22.28)^{*}$ & $33.00(22.64)^{*}$ & $27.83(23.28)^{*}$ & 0.524 \\
PHQ-9 & $16.92(6.87)$ & $9.70(7.92)^{*}$ & $9.57(8.51)^{*}$ & $8.83(9.14)^{*}$ & 0.537 \\
GAF & $54.24(6.33)$ & $63.30(9.20)^{*}$ & $65.86(10.53)^{*}$ & $71.06(12.04)^{*}$ & 0.575 \\
\hline
\end{tabular}

${ }^{*} \mathrm{p}<0.001$, $\mathrm{p}$ values refer to comparisons from baseline to T2, T3, and T4, respectively. CAPS: Clinician Administered PTSD Scale for DSM-5, PCL-5: PTSD Check List-5, PHQ-9: Patient Health Questionnaire-9, GAF: Global Assessment of Function, PTSD: post-traumatic stress disorder, SD: standard deviation

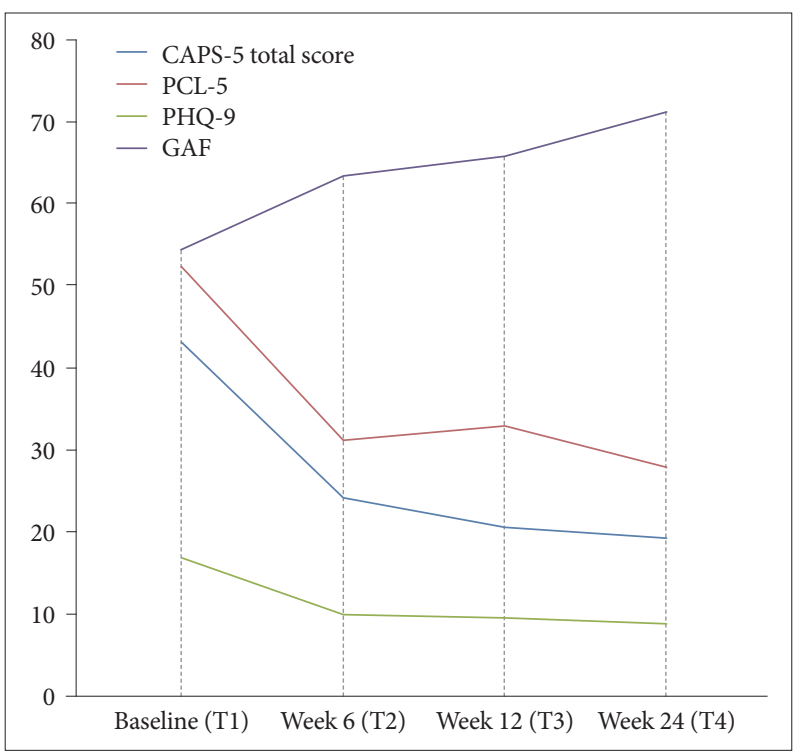

Figure 2. Changes in primary and other measures. T1: baseline, T2: 6 weeks follow-up, T3: 12 weeks follow-up, T4: 24 weeks follow-up. CAPS: Clinician Administered PTSD Scale for DSM-5, PCL-5: PTSD Check List-5, PHQ-9: Patient Health Questionnaire-9, GAF: Global Assessment of Function.

All scores were significantly reduced at each follow-up assessment compared to those at baseline (Table 2). The rmANOVA results indicated that the scores of CAPS ( $\mathrm{F} 40.278, \mathrm{p}<0.001$ ), GAF (F 22.989, p<0.001), PCL-5 (F 17.611, p<0.001), and PHQ-9 (F 18.530, p $<0.001$ ) significantly reduced over time (Figure 2) and yielded large effect size (CAPS 0.703, PCL-5 0.524, PHQ9 0.537, GAF 0.575).

There were no significant differences between participants who did not satisfy the PTSD diagnosis and those who did according to demographic and clinical characteristics, except for baseline symptom scores. There was no difference in PTSD duration between the two groups (improved:non-improved group (mean $\pm S D)$; at 6-week 35.36 $\pm 39.16: 52.67 \pm 42.00$, p= 0.439 , at 12 -week $43.53 \pm 40.55: 43.43 \pm 44.19, \mathrm{p}=0.891$, at 24 week 41.63 $\pm 11.13: 45.25 \pm 45.06, \mathrm{p}=0.959$ ). There were significant differences in the PCL-5, PHQ-9, and CAPS scores at baseline between the two groups in the 6 weeks follow-up assessment (Table 3). There was a significant difference in the diagnosis of comorbid depression at baseline between the two groups in the follow-up assessments at 6 and 12 weeks. At the 12 weeks follow-up assessment, the participants who did not meet the PTSD diagnosis had higher satisfaction after treatment than those who still met the PTSD diagnosis. However, these differences were not statistically significant at 24 weeks.

\section{DISCUSSION}

This study aimed to explore the efficacy of WET among a Korean sample presenting with clinically diagnosed PTSD. Our findings demonstrate that Korean participants with PTSD benefitted significantly from a brief written exposure treatment. Specifically, self-reported and clinician-assessed PTSD symptoms, depressive symptoms, and overall functioning improved after participants underwent WET.

Numerous studies have demonstrated reductions in PTSD and related psychopathologies with exposure therapy. ${ }^{15,16,20,33}$ However, global functioning is another measure of symptom reduction. Improvements in PTSD with exposure therapy were associated with improvements in psychosocial functioning. ${ }^{34,35}$ Therefore, the reduction of PTSD symptoms through WET was expected to improve general functioning. Previous research has not reported on the improvement of functioning through WET; therefore, it is significant that the improvement of general functioning after WET was identified in this study.

We found that the treatment effect of WET was maintained at week 24. At the 12 and 24 week follow-up, 4 and 2 additional participants respectively showed improvements in PTSD. Several theories explain the mechanisms for the effects of WET. Traditionally, the emotional processing theory ${ }^{36}$ and cognitive models of PTSD ${ }^{37}$ underpin EBT, such as PE and CPT. Thus, WET may reduce PTSD and other symptoms through habituation during five sessions; specifically, by helping individuals more effectively organize and integrate their traumatic memories. ${ }^{14}$ Inhibitory learning, a recently supported mechanism for exposure therapy, may also be an important mechanism for this treatment effect and maintaining the effects of WET. Inhibitory learning through EBT, including WET, provide the op- 
Table 3. Comparison between participants who did not satisfy the PTSD diagnosis after 6 and 12 weeks and those who did

\begin{tabular}{|c|c|c|c|c|c|c|}
\hline & \multicolumn{3}{|c|}{6 weeks follow-up (N=23) } & \multicolumn{3}{|c|}{12 weeks follow-up $(\mathrm{N}=22)$} \\
\hline & $\begin{array}{c}\text { PTSD (-) } \\
\mathrm{N}=14\end{array}$ & $\begin{array}{c}\operatorname{PTSD}(+) \\
\mathrm{N}=9\end{array}$ & $\mathrm{p}$ & $\begin{array}{c}\text { PTSD (-) } \\
\mathrm{N}=15\end{array}$ & $\begin{array}{c}\text { PTSD (+) } \\
\mathrm{N}=7\end{array}$ & $\mathrm{p}$ \\
\hline \multicolumn{7}{|l|}{ Baseline score } \\
\hline CAPS & $40.9(8)$ & $51.3(9.9)$ & 0.011 & $44.0(7.56)$ & $47.57(14.93)$ & 0.459 \\
\hline GAF & $53.6(5.08)$ & $50.1(5.53)$ & 0.131 & $52.5(2.92)$ & $50.7(8.81)$ & 0.471 \\
\hline PCL-5 & $50.9(11.93)$ & $65(12.9)$ & 0.014 & $52.9(11.89)$ & $62.7(17.12)$ & 0.131 \\
\hline PHQ-9 & $14.3(4.97)$ & $22.7(4.95)$ & 0.001 & $16.3(5.83)$ & $20.9(7.20)$ & 0.126 \\
\hline Diagnosis of comorbid depression at baseline & $2(14.3)$ & $6(66.7)$ & 0.023 & $3(20.0)$ & $5(71.4)$ & 0.052 \\
\hline Treatment expectation & $0.4(4.62)$ & $0.4(2.47)$ & 0.966 & $0(4.87)$ & $0.5(2.16)$ & 0.789 \\
\hline Treatment satisfaction & $27.8(3.38)$ & $26.9(3.44)$ & 0.388 & $28.3(3.22)$ & $25.1(2.79)$ & 0.036 \\
\hline
\end{tabular}

The results at 24 week follow up were not statistically significant. CAPS: Clinician Administered PTSD Scale for DSM-5, PCL-5: PTSD Check List-5, PHQ-9: Patient Health Questionnaire-9, GAF: Global Assessment of Function, PTSD: post-traumatic stress disorder

portunity to be instilled with a sense of unconditional learned safety. ${ }^{38}$ It is expected that patients will continue to utilize concepts learned from exposure therapy in daily life after the end of formal therapy sessions. ${ }^{38}$

We analyzed the difference between the two groups according to treatment outcome at each follow up assessment. Sociodemographic factors, trauma-related variables such as age at the time of trauma exposure, and trauma type were not different between two groups. These results are similar to those of previous studies. Although benzodiazepine use is associated with more symptoms at follow-up after $\mathrm{PE},{ }^{13,17,39}$ our findings did not show an association between benzodiazepine use and treatment outcome.

The results of this study demonstrate that WET was well-tolerated and well-received by Korean participants. The dropout rates during WET in Western studies was around 6\%-9\%. ${ }^{14,15}$ Similarly, the dropout rate in this study was $8 \%$. However, it is evidently lower compared to the $24 \%$ to $36 \%$ drop-out rates reported in previous PE or CPT studies. ${ }^{40,41}$

Despite recent treatment guidelines for PTSD, which recommend exposure therapy as a first-line treatment, the issues of Korean mental health care services, including low medical fees for psychotherapy, quantity-oriented hospital evaluations, and heavy outpatient workload ${ }^{42}$ are barrier to conduct EBTs for PTSD in Korea. Thus, we suggest that the brevity and tolerability of WET is an efficient and effective alternative therapy in the Korean medical system.

This study is meaningful as it is the first Asian study performed and evaluated by certified professions to prove the effects of WET. In addition, in this study, WET was implemented for patients with clinically diagnosed PTSD, and the effectiveness of treatment was evaluated by clinical evaluators. However, there are several limitations to our study. First, our experiment was an uncontrolled trial and we only analyzed completer data. There- fore, our findings, though significant, could not be considered conclusive. Second, suicidal patients with present suicidal ideation severe enough to warrant immediate intervention were excluded from the present study. Therefore, it should be noted that these conclusions should not be generalized to PTSD patients that present suicidal intent. Third, all sessions were served by only one therapist. It is reported that the therapist effects in naturalistic studies averaged $5 \%{ }^{43}$ and the effects of therapist effect cannot be completely excluded in this study. Fourth, medication effect might contribute to the significant reduction of symptoms after WET in this study. To minimize the effect of pharmacotherapy, all participants maintained the dose of medication at least 8 weeks before the first session. During the 5 sessions, medication dose was maintained. In addition, no subjects increased their medication dose in the follow-up period. Finally, the follow-up period was not enough long, therefore the long-term treatment effects of WET could not be confirmed in this study. In the future, long-term follow-up research on Asian samples is needed to obtain conclusive results.

In conclusion, this study suggests the feasibility of implementing WET for various types of patients with PTSD in Korea and other Asian countries. This will improve not only PTSD symptoms, but also depressive symptoms and general functioning. Future large-scale randomized controlled trials investigating the effectiveness of WET in the Asian population are necessary.

\section{Availability of Data and Material}

The datasets generated or analyzed during the study are available from the corresponding author on reasonable request.

\section{Conflicts of Interest}

The authors have no potential conflicts of interest to disclose.

\section{Author Contributions}

Conceptualization: Kyeong-Sook Choi, Je-Chun Yu, Ji-Ae Yun. Formal analysis: Ju-Eun Park, Yu-Ri Han. Investigation: Jeong-Eun Kim, Yu-Ri 
Han, Ji-Ae Yun. Methodology: Ji-Ae Yun. Software: Yu-Ri Han. Supervision: Ji-Ae Yun. Visualization: Ju-Eun Park. Writing_original draft: JuEun Park, Ji-Ae Yun. Writing_review \& editing: Kyeong-Sook Choi.

\section{ORCID iDs}

$\begin{array}{ll}\text { Ju-Eun Park } & \text { https://orcid.org/0000-0002-3861-832X } \\ \text { Kyeong-Sook Choi } & \text { https://orcid.org/0000-0003-3933-7121 } \\ \text { Yuri Han } & \text { https://orcid.org/0000-0002-4618-2546 } \\ \text { Jeong-Eun Kim } & \text { https://orcid.org/0000-0003-0612-2335 } \\ \text { Jay Song } & \text { https://orcid.org/0000-0003-1925-2489 } \\ \text { Je-Chun Yu } & \text { https://orcid.org/0000-0003-1665-0289 } \\ \text { Ji-Ae Yun } & \text { https://orcid.org/0000-0002-6682-3275 }\end{array}$

\section{Funding Statement}

This research was supported by EMBRI Grants 2019-EMBRI-DJ0003 from the Eulji University.

\section{REFERENCES}

1. Sadock BJ, Sadock VA, Ruiz P. Kaplan \& Sadock's Synopsis of Psychiatry: Behavioral Sciences/Clinical Psychiatry. Philadelphia: Wolters Kluwer; 2015.

2. Vogt D, Smith BN, Fox AB, Amoroso T, Taverna E, Schnurr PP. Consequences of PTSD for the work and family quality of life of female and male U.S. Afghanistan and Iraq War veterans. Soc Psychiatry Psychiatr Epidemiol 2017;52:341-352.

3. Savoca E, Rosenheck R. The civilian labor market experiences of Vietnam-era veterans: the influence of psychiatric disorders. J Ment Health Policy Econ 2000;3:199-207.

4. Sripada RK, Henry J, Yosef M, Levine DS, Bohnert KM, Miller E, et al. Occupational functioning and employment services use among VA primary care patients with posttraumatic stress disorder. Psychol Trauma 2018;10:140-143.

5. Lambert JE, Engh R, Hasbun A, Holzer J. Impact of posttraumatic stress disorder on the relationship quality and psychological distress of intimate partners: a meta-analytic review. J Fam Psychol 2012;26:729737.

6. Taft CT, Watkins LE, Stafford J, Street AE, Monson CM. Posttraumatic stress disorder and intimate relationship problems: a meta-analysis. J Consult Clin Psychol 2011;79:22-33.

7. Fox R, Hyland P, McHugh Power J, Coogan AN. Patterns of comorbidity associated with ICD-11 PTSD among older adults in the United States. Psychiatry Res 2020;290:113171.

8. Pacella ML, Hruska B, Delahanty DL. The physical health consequences of PTSD and PTSD symptoms: a meta-analytic review. J Anxiety Disord 2013;27:33-46.

9. Hamblen JL, Norman SB, Sonis JH, Phelps AJ, Bisson JI, Nunes VD, et al. A guide to guidelines for the treatment of posttraumatic stress disorder in adults: an update. Psychotherapy (Chic) 2019;56:359-373.

10. Sloan DM, Marx BP. Written Exposure Therapy for PTSD: A Brief Treatment Approach for Mental Health Professionals. Washington, DC: American Psychological Association; 2019.

11. McLean CP, Asnaani A, Foa EB. Prolonged Exposure Therapy. In: Schnyder U, Cloitre M, Editors. Evidence Based Treatments for Trauma-Related Psychological Disorders: A Practical Guide for Clinicians. Cham: Springer International Publishing, 2015, p.143-159.

12. Resick PA, Monson CM, Chard KM. Cognitive Processing Therapy for PTSD: A Comprehensive Manual. New York: Guilford Publications; 2016.

13. Thompson-Hollands J, Marx BP, Sloan DM. Brief novel therapies for PTSD: Written Exposure Therapy. Curr Treat Options Psychiatry 2019; 6:99-106.

14. Sloan DM, Marx BP, Bovin MJ, Feinstein BA, Gallagher MW. Written exposure as an intervention for PTSD: a randomized clinical trial with motor vehicle accident survivors. Behav Res Ther 2012;50:627-635.
15. Sloan DM, Marx BP, Lee DJ, Resick PA. A brief exposure-based treatment vs cognitive processing therapy for posttraumatic stress disorder: a randomized noninferiority clinical trial. JAMA Psychiatry 2018;75: 233-239.

16. Thompson-Hollands J, Marx BP, Lee DJ, Resick PA, Sloan DM. Longterm treatment gains of a brief exposure-based treatment for PTSD. Depress. Anxiety 2018;35:985-991.

17. Marx BP, Thompson-Hollands J, Lee DJ, Resick PA, Sloan DM. Estimated intelligence moderates cognitive processing therapy outcome for posttraumatic stress symptoms. Behav Ther 2021;52:162-169.

18. Kawakami N, Tsuchiya M, Umeda M, Koenen KC, Kessler RC. Trauma and posttraumatic stress disorder in Japan: results from the World Mental Health Japan Survey. J Psychiatr Res 2014;53:157-165.

19. Asukai N, Saito A, Tsuruta N, Kishimoto J, Nishikawa T. Efficacy of exposure therapy for Japanese patients with posttraumatic stress disorder due to mixed traumatic events: a randomized controlled study. J Trauma Stress 2010;23:744-750.

20. Asukai N, Saito A, Tsuruta N, Ogami R, Kishimoto J. Pilot study on prolonged exposure of Japanese patients with posttraumatic stress disorder due to mixed traumatic events. J Trauma Stress 2008;21:340-343.

21. Schnyder U, Bryant RA, Ehlers A, Foa EB, Hasan A, Mwiti G, et al. Culture-sensitive psychotraumatology. Euro J Psychotraumatol 2016;7: 31179.

22. Kessler RC, Aguilar-Gaxiola S, Alonso J, Benjet C, Bromet EJ, Cardoso G, et al. Trauma and PTSD in the WHO World Mental Health Surveys. Eur J Psychotraumatol 2017;8:1353383.

23. Weathers FW, Bovin MJ, Lee DJ, Sloan DM, Schnurr PP, Kaloupek DG, et al. The Clinician-Administered PTSD Scale for DSM-5 (CAPS5): development and initial psychometric evaluation in military veterans. Psychol Assess 2018;30:383-395.

24. Kim WH, Jung YE, Roh D, Kim D, Kang SH, Chae JH, et al. Reliability and validity of the Korean version of Clinician-Administered Posttraumatic Stress Disorder Scale for DSM-5. J Korean Med Sci 2019;34: e219.

25. Weathers FW, Litz BT, Keane TM, Palmieri PA, Marx BP, Schnurr PP. The PTSD checklist for DSM-5 (PCL-5). 2013. Scale from the National Center for PTSD. Available at: www.ptsd.va.gov. 2014.

26. Oh SI, Won SD, Lee SH, Yoo SY, Kim HC, Kim HJ. Reliability and validity of the Korean version of the PTSD checklist civilian version in North Korean defectors. J Korean Neuropsychiatr Assoc 2014;53:410417.

27. Choi HS, Choi JH, Park KH, Joo KJ, Ga H, Ko HJ, et al. Standardization of the Korean version of Patient Health Questionnaire-9 as a screening instrument for major depressive disorder. J Korean Acad Fam Med 2007;28:114-119.

28. American Psychiatric Association. Diagnostic and Statistical Manual of Mental Disorders, 4th Edition, Text Revision (DSM-IV-TR). Washington DC: American Psychiatric Publishing; 2000.

29. Sloan DM, Lee DJ, Litwack SD, Sawyer AT, Marx BP. Written exposure therapy for veterans diagnosed with PTSD: a pilot study. J Trauma Stress 2013;26:776-779.

30. Borkovec TD, Nau SD. Credibility of analogue therapy rationales. J Behav Ther Exp Psychiatry 1972;3:257-260.

31. Devilly GJ, Borkovec TD. Psychometric properties of the credibility/ expectancy questionnaire. J Behav Ther Exp Psychiatry 2000;31:73-86.

32. Larsen DL, Attkisson CC, Hargreaves WA, Nguyen TD. Assessment of client/patient satisfaction: development of a general scale. Eval Program Plann 1979;2:197-207.

33. Hori A, Takebayashi Y, Tsubokura M, Kim Y. Efficacy of prolonged exposure therapy for a patient with late-onset PTSD affected by evacuation due to the Fukushima nuclear power plant accident. BMJ Case Rep 2019;12:e231960.

34. Reich K, Nemeth LS, Mueller M, Sternke LM, Acierno R. Does psychosocial functioning improve with prolonged exposure in veterans with PTSD?: exploring traditional and home-based telehealth delivery 
methods. J Psychosoc Nurs Ment Health Serv 2021;59:31-40.

35. Rauch SA, Grunfeld TE, Yadin E, Cahill SP, Hembree E, Foa EB. Changes in reported physical health symptoms and social function with prolonged exposure therapy for chronic posttraumatic stress disorder. Depress Anxiety 2009;26:732-738.

36. Foa EB, Kozak MJ. Emotional processing of fear. Exposure to corrective information. Psychol Bull 1986;99:20-35.

37. Ehlers A, Clark DM. A cognitive model of posttraumatic stress disorder. Behav Res Ther 2000;38:319-345.

38. Abramowitz JS, Deacon BJ, Whiteside SPH. Exposure Therapy for Anxiety, Second Edition: Principles and Practice. New York: Guilford Publications; 2019.

39. van Minnen A, Arntz A Keijsers GP. Prolonged exposure in patients with chronic PTSD: predictors of treatment outcome and dropout. Behav Res Ther 2002;40:439-457.

40. Kline AC, Cooper AA, Rytwinksi NK, Feeny NC. Long-term efficacy of psychotherapy for posttraumatic stress disorder: a meta-analysis of randomized controlled trials. Clin Psychol Rev 2018;59:30-40.

41. Imel ZE, Laska K, Jakupcak M, Simpson TL. Meta-analysis of dropout in treatments for posttraumatic stress disorder. J Consult Clin Psychol 2013;81:394-404.

42. Park JI, Oh KY, Chung YC. Psychiatry in Korea. Asian J Psychiatr 2013; 6:186-190.

43. Johns RG, Barkham M, Kellett S, Saxon D. A systematic review of therapist effects: a critical narrative update and refinement to Baldwin and Imel's (2013) review. Clin Psychol Rev 2019;67:78-93. 